\title{
Formal First Integrals of General Dynamical Systems
}

\author{
Jia Jiao, ${ }^{1}$ Wenlei Li, ${ }^{2}$ and Qingjian $Z_{h o u}{ }^{1}$ \\ ${ }^{1}$ College of Science, Dalian Minzu University, Dalian 116600, China \\ ${ }^{2}$ School of Mathematics, Jilin University, Changchun 130012, China \\ Correspondence should be addressed to Wenlei Li; lwlei@jlu.edu.cn
}

Received 22 February 2016; Accepted 4 July 2016

Academic Editor: Andrei D. Mironov

Copyright (C) 2016 Jia Jiao et al. This is an open access article distributed under the Creative Commons Attribution License, which permits unrestricted use, distribution, and reproduction in any medium, provided the original work is properly cited.

\begin{abstract}
The goal of this paper is trying to make a complete study on the integrability for general analytic nonlinear systems by first integrals. We will firstly give an exhaustive discussion on analytic planar systems. Then a class of higher dimensional systems with invariant manifolds will be considered; we will develop several criteria for existence of formal integrals and give some applications to illustrate our results at last.
\end{abstract}

\section{Introduction}

Investigating the existence of first integrals is well known as an important problem in the study of nonlinear systems arising in applied mathematics and physics [1]. In this paper, we will mainly consider the related topics for the general dynamical system,

$$
\begin{aligned}
& \dot{z}=u(z), \\
& z=\left(z_{1}, \ldots, z_{n}\right) \in \mathbb{C}^{n},
\end{aligned}
$$

with $n$-dimensional analytic vector-valued function $u(z)$ satisfying $u(0)=0$. A function $\Phi: U \rightarrow \mathbb{C}(U$ an open set) is said to be a first integral of (1), if $\Phi(z)$ is a constant along any solution curve of system (1). In general, if system (1) has sufficient first integrals, such that the general solution can be expressed by quadrature of these integrals, we say it is integrable.

Remark 1. (1) It is well known that $n$-dimensional autonomous system (1) with $n-1$ functionally independent first integrals on $U$ is integrable as the corresponding solutions can be solved by implicit function theorem. Especially for planar systems, the existence of first integral is, in fact, equivalent to integrability.
(2) If a first integral $\Phi(z)$ of (1) is differentiable on $U$, it obviously satisfies

$$
\left\langle\nabla_{z} \Phi(z), u(z)\right\rangle \equiv 0,
$$

where $\nabla_{z}$ denotes the gradient with respect to $z$ and $\langle\cdot, \cdot\rangle$ denotes the classical Euclidian inner product. If $\Phi(z)$ can be expanded in formal series in a neighborhood of the fixed point $z=0$, we call it a formal first integral.

One of the effective approaches to investigate the integrability of system (1) is using normal form theory with the resonance condition of corresponding linear part [2] to find sufficient many necessary conditions for the existence of first integrals. The related idea, as far as we know, can be traced to the work of Poincaré; he found that [3] if the Jacobian matrix $A=D u(0)$ is diagonal and the eigenvalues $\lambda_{1}, \ldots, \lambda_{n}$ of it are $\mathbb{N}$-independent, then system (1) does not have any formal first integral in a neighborhood of the origin $z=0$; that is, they do not satisfy any resonant condition of the form

$$
\begin{aligned}
\sum_{j=1}^{n} k_{j} \lambda_{j} & =0, \quad k_{j} \in \mathbb{N} \cup\{0\}, \\
\sum_{j=1}^{n} k_{j} & \geq 1 .
\end{aligned}
$$


Following Poincaré's work, a lot of results have been obtained on the nonintegrability and partial integrability of general nonlinear dynamical systems; see [2, 4-8], for example. In 1996, Furta [2] provided an elementary proof of Poincaré's result; moreover, by using singular analysis, he also gave some valuable study of the nonexistence and partial existence of analytic first integrals for semiquasihomogeneous systems. Recently, Shi and Li [9] extended Furta's results to study the case that $A$ is not necessarily a diagonal matrix and the case that eigenvalues of $A$ are resonant [10-12]. So far, the above results have been proved to be very useful and effective from a large number of applications [13], while there are very few works using these known results to give a systematic investigation of the integrability of general dynamical systems rather than some specific examples. The main goal of the present paper is trying to make a complete discussion of this topic. As a beginning, let us have a look at the existence of formal integrals to planar system

$$
\dot{x}=A x+F(x), \quad x \in \mathbb{C}^{2},
$$

where $A$ is a $2 \times 2$ complex matrix and $F(x)=O\left(|x|^{2}\right)$ is analytic in $x$. Without loss of generality, we also assume $A$ is in Jordan form. Then,

(1) if $A=\left(\begin{array}{cc}\lambda_{1} & 0 \\ 0 & \lambda_{2}\end{array}\right)$, with $\lambda_{1}, \lambda_{2}$ not satisfying (3), by the Poincaré's theorem, system (4) is not formally integrable;

(2) if $A=\left(\begin{array}{cc}\lambda_{1} & 0 \\ 0 & \lambda_{2}\end{array}\right)$, with $\lambda_{1}, \lambda_{2}$ satisfying (3) and $\lambda_{1} \lambda_{2} \neq 0$, by the results in [10], system (4) is formally integrable if and only if one of the equations in the normal form of the transformed system from (4) by transformation $(u, v)=\left(x_{1}^{k_{1}} x_{2}^{k_{2}}, x_{2}\right)$ is trivial;

(3) if $A=\left(\begin{array}{ll}\lambda & 1 \\ 0 & \lambda\end{array}\right)$ with $\lambda \neq 0$, by the results in [9], system (4) is not formally integrable.

And there remain two cases needing more investigations.

Case 1. Consider $A=\left(\begin{array}{ll}0 & 1 \\ 0 & 0\end{array}\right)$.

Case 2. Consider $A=\left(\begin{array}{ll}0 & 0 \\ 0 & 0\end{array}\right)$.

Our first main work in the paper is to give an exhaustive discussion of the previous two cases for general planar systems. Then we will go ahead with some discussions for a class of higher dimensional systems,

$$
\begin{aligned}
& \dot{x}=F(x, y), \\
& \dot{y}=G(x, y),
\end{aligned}
$$

$$
(x, y) \in \mathbb{R}^{d} \times \mathbb{R}^{s}
$$

with an invariant manifold expressed as

$$
\mathscr{Z}=\left\{(x, y) \mid y=h(x), x \in U \subset \mathbb{R}^{d}\right\},
$$

where $F(x, y), G(x, y)$, and $h(x)$ are analytic vector-valued functions, $F(0,0)=0, G(0,0)=0$. We especially will combine the center manifold theory [14] and the known results by Shi and $\mathrm{Li}$ [9] to give several necessary conditions of integrability results for general dynamical systems with center manifolds.

This paper is organized as follows. In Section 2, we will give an exhaustive discussion of Cases 1 and 2 for general planar systems. In Section 3, we will give a new method to investigate integrability problem of general dynamical systems with center manifolds, and several examples are presented as applications of our results at last.

\section{Integrability and Nonintegrability of Planar Systems}

2.1. Case 1. By the normal form theory [14], system (4) can be transformed into

$$
\dot{x}=A x+F(x), \quad x \in \mathbb{C}^{2},
$$

with

$$
\begin{aligned}
A & =\left(\begin{array}{ll}
0 & 1 \\
0 & 0
\end{array}\right), \\
F(x) & =\left(\begin{array}{l}
F_{1}(x) \\
F_{2}(x)
\end{array}\right)=\left(\begin{array}{c}
\sum_{k=2}^{\infty} a_{k, 1} x_{1}^{k} \\
\sum_{k=2}^{\infty} a_{k, 2} x_{1}^{k}
\end{array}\right),
\end{aligned}
$$

with constants $a_{k, j} \in \mathbb{C}, j=1,2, k=2,3, \ldots$. Then, we have the following statement.

Theorem 2. System (7) is formally integrable if and only if $F_{1}(x) F_{2}(x) \equiv 0$.

Proof. The proof will be divided into three cases.

(1) If $F_{1}(x) \equiv 0$, then (7) is a classical complex Hamiltonian system with 1 degree of freedom, which implies the integrability of itself.

(2) If $F_{2}(x) \equiv 0$, then (7) has a nontrivial integral $I(x)=$ $x_{2}$, which also leads to the integrability.

(3) If $F_{1}(x) F_{2}(x) \not \equiv 0$, then there must exist two integers $n, m \in \mathbb{N}$, such that $n \geq 2, m \geq 2$, and $a_{n, 1} \neq 0$, $a_{m, 2} \neq 0$ while $a_{i, 1}=0, a_{j, 2}=0$ when $i<n, j<m$. Assume $I(x)=\sum_{k=l}^{\infty} I_{k}(x)$ is a first integral of system (7); here $I_{k}(x)$ is the $k$ th order homogeneous terms of $I(x), k=l, l+1, \ldots$. Then by the definition of first integral

$$
\left\langle\nabla_{x} I(x), A x+F(x)\right\rangle \equiv 0 .
$$


Equating the same order terms in $x$, we have

$$
\begin{aligned}
& O(l): \partial_{x_{1}} I_{l}(x) x_{2} \equiv 0 ; \\
& O(l+1): \partial_{x_{1}} I_{l+1}(x) x_{2} \\
& =-\left(a_{21} \partial_{x_{1}} I_{l}(x)+a_{22} \partial_{x_{2}} I_{l}(x)\right) x_{1}^{2} ; \\
& \vdots \\
& O(k+1): \partial_{x_{1}} I_{k+1}(x) x_{2} \\
& =-\sum_{i=l}^{k}\left(a_{k+2-i, 1} \partial_{x_{1}} I_{i}(x)+a_{k+2-i, 2} \partial_{x_{2}} I_{i}(x)\right) x_{1}^{k+2-i} \\
& \quad=\sum_{j=0}^{k+1} C_{k+1, j} x_{1}^{j} x_{2}^{k+1-j} ;
\end{aligned}
$$

where complex constants $C_{k+1, j}, k=l, l+1, \ldots, j=$ $0, \ldots, k+1$, are coefficients of the polynomials in the right hand side of (10). Furthermore, by direct computation, one can find that there exists a number $k(\geq l)$ such that $C_{l, l}=$ $\cdots=C_{k, k} \equiv 0$ and $C_{k+1, k+1}$ can be represented as a nontrivial polynomial of $\left(a_{n, 1}, a_{m, 2}\right)$; that is, $C_{k+1, k+1}=$ $C_{k+1, k+1}\left(a_{n, 1}, a_{m, 2}\right)$.

Then if the parameters $\left(a_{n, 1}, a_{m, 2}\right)$ are chosen such that $C_{k+1, k+1}\left(a_{n, 1}, a_{m, 2}\right) \neq 0$, (10) does not hold; this implies the nonexistence of nontrivial first integrals. In general, making the change of variables $x_{1}=\sigma_{1} y_{1}, x_{2}=\sigma_{2} y_{2}, t=$ $\left(\sigma_{1} / \sigma_{2}\right) s$ with nonzero parameters $\sigma_{1}, \sigma_{2}$ to be fixed, (7) can be equivalently transformed into

$$
y^{\prime}=A y+G(y), \quad y \in \mathbb{C}^{2},
$$

with

$$
G(y)=\left(\begin{array}{l}
G_{1}(y) \\
G_{2}(y)
\end{array}\right)=\left(\begin{array}{c}
\sum_{k=n}^{\infty} b_{k, 1} y_{1}^{k} \\
\sum_{k=m}^{\infty} b_{k, 2} y_{1}^{k}
\end{array}\right)
$$

with constants $b_{k, j} \in \mathbb{C}$, and

$$
\begin{aligned}
& b_{n, 1}=\frac{\sigma_{1}^{n}}{\sigma_{2}} a_{n, 1}, \\
& b_{m, 2}=\frac{\sigma_{1}^{m+1}}{\sigma_{2}^{2}} a_{m, 2} .
\end{aligned}
$$

Then, if $m \neq 2 n-1$, one can conclude from the above two equations that, for any $\left(b_{n, 1}, b_{m, 2}\right) \in \mathbb{C}^{2}$ such that $b_{n, 1} b_{m, 2} \neq 0$, the parameters $\sigma_{1}, \sigma_{2}$ exist, which implies the nonexistence of first integrals for (11) and (7).

If $m=2 n-1$, then (13) becomes $b_{n, 1}^{2} a_{2 n-1,2}=b_{2 n-1,2} a_{n, 1}^{2}$. And we can choose two parameters $\sigma_{1}, \sigma_{2}$ to make $b_{n, 1}$ a transcendental number; consequently, $b_{2 n-1,2}$ and $\delta=$ $b_{2 n-1,2} / b_{n, 1}$ are also transcendental numbers. Now assume system (11) has a first integral $H(y)=\sum_{k=0}^{\infty} H_{k}\left(y_{1}\right) y_{2}^{k}$; here $H_{k}\left(y_{1}\right)$ is a formal function of $y_{1}, k=l, l+1, \ldots$. Then, by the definition of first integral, we have

$$
0 \equiv\left\langle\nabla_{y} H(y), A y+G(y)\right\rangle .
$$

Equating the same order terms with respect to $y_{2}$, we have

$$
\begin{aligned}
& O\left(y_{2}^{0}\right): H_{0}^{\prime}\left(y_{1}\right)\left(b_{n, 1} y_{1}^{n}+\cdots\right) \\
& =-H_{1}\left(y_{1}\right)\left(b_{2 n-1,2} y_{1}^{2 n-1}+\cdots\right) \\
& O\left(y_{2}\right): H_{0}^{\prime}\left(y_{1}\right)=H_{1}^{\prime}\left(y_{1}\right)\left(b_{n, 1} y_{1}^{n}+\cdots\right) \\
& -2 H_{2}\left(y_{1}\right)\left(b_{2 n-1,2} y_{1}^{2 n-1}+\cdots\right) ; \\
& \vdots \\
& O\left(y_{2}^{k+1}\right): H_{k}^{\prime}\left(y_{1}\right)=H_{k+1}^{\prime}\left(y_{1}\right)\left(b_{n, 1} y_{1}^{n}+\cdots\right) \\
& -(k+2) H_{k+2}\left(y_{1}\right)\left(b_{2 n-1,2} y_{1}^{2 n-1}+\cdots\right) ;
\end{aligned}
$$

Firstly, from the third general equation, it is not difficult to conclude that the lowest order of $H_{k}^{\prime}\left(y_{1}\right)$ is at least $2 n-1, k=$ $0,1, \ldots$. Substituting this conclusion into the first equation, we have that the lowest orders of $H_{0}^{\prime}\left(y_{1}\right)$ and $H_{1}\left(y_{1}\right)$ are at least $3 n-1$ and $2 n$, respectively. Repeatedly, considering the third general equation, we can get that, in fact, the lowest orders of $H_{k}^{\prime}\left(y_{1}\right)$ and $H_{k}\left(y_{1}\right)$ are at least $3 n-1$ and $3 n$. Finally, one can easily conclude that $H_{k}\left(y_{1}\right) \equiv 0, k=1,2, \ldots$. However, from the first equation, in order to make $H(y)$ nontrivial, it is necessary to let $H_{0}^{\prime}\left(y_{1}\right) H_{1}\left(y_{1}\right) \neq 0$, which contradicts to the previous discussions. This completes the whole proof.

2.2. Case 2. In this case, it is not difficult to see that if system (4) is formally integrable, then so is the homogeneous subsystem

$$
\dot{x}=F_{2}(x), \quad x \in \mathbb{C}^{2},
$$

where $F_{2}(x)$ is the second-order homogeneous terms of $F(x)$. Without loss of generality, we expand it as

$$
\begin{aligned}
F_{2}(x) & =\left(\begin{array}{l}
a_{1} x_{1}^{2}+b_{1} x_{1} x_{2}+c_{1} x_{2}^{2} \\
a_{2} x_{2}^{2}+b_{2} x_{1} x_{2}+c_{2} x_{1}^{2}
\end{array}\right), \\
\Xi & =\left(\begin{array}{lll}
a_{1} & b_{1} & c_{1} \\
c_{2} & b_{2} & a_{2}
\end{array}\right)
\end{aligned}
$$

with $a_{i}, b_{i}, c_{i} \in \mathbb{C}, i=1,2$; then we have the following results.

Theorem 3. (1) If $\operatorname{Rank}(\Xi) \leq 1,(16)$ is integrable.

(2) If $\operatorname{Rank}(\Xi)=2$ and $c_{1}=a_{2}=0$, (16) is formally integrable if and only if two eigenvalues of the matrix $\left(\begin{array}{ll}a_{1} & b_{1} \\ c_{2} & b_{2}\end{array}\right)$ satisfy the resonant condition (3). 
(3) If $\operatorname{Rank}(\Xi)=2, c_{1}=0, a_{2} \neq 0, a_{2}=b_{1}$, and $a_{1}=b_{2}$, (16) is not formally integrable.

(4) If $\operatorname{Rank}(\Xi)=2, c_{1}=0, a_{2} \neq 0$, and $\left(a_{2}-b_{1}\right)^{2}+\left(a_{1}-\right.$ $\left.b_{2}\right)^{2} \neq 0$, then, (16) is formally integrable if and only if there exist three integers $k_{1}, k_{2}, l$, such that $0<k_{1} \leq k_{2} \leq l, k_{1} b_{1}+$ $\left(l-k_{1}\right) a_{2}=0$, and $k_{2} a_{1}+\left(l-k_{2}\right) b_{2}=0$.

Remark 4. By the same status of the variables $x_{1}$ and $x_{2}$, Theorem 3 gives a complete illustration of the problem of the integrability for system (16). In fact, if $c_{1} \neq 0$, one can make a change of variables

$$
\left(\begin{array}{l}
y_{1} \\
y_{2}
\end{array}\right)=\left(\begin{array}{ll}
1 & 0 \\
\sigma & 1
\end{array}\right)\left(\begin{array}{l}
x_{1} \\
x_{2}
\end{array}\right)
$$

with a number $\sigma$ to be fixed later, such that (16) can be equivalently transformed into

$$
\begin{aligned}
& \dot{y}_{1}=\widetilde{a}_{1} y_{1}^{2}+\widetilde{b}_{1} y_{1} y_{2}+\widetilde{c}_{1} y_{2}^{2}, \\
& \dot{y}_{2}=\widetilde{a}_{2} y_{2}^{2}+\widetilde{b}_{2} y_{1} y_{2}+\widetilde{c}_{2} y_{1}^{2},
\end{aligned}
$$

with

$$
\begin{aligned}
& \widetilde{a}_{1}=a_{1}-\sigma b_{1}+\sigma^{2} c_{1}, \\
& \widetilde{b}_{1}=b_{1}-2 \sigma c_{1}, \\
& \widetilde{c}_{1}=c_{1}, \\
& \widetilde{a}_{2}=a_{2}+\sigma c_{1}, \\
& \widetilde{b}_{2}=b_{2}+\sigma\left(b_{1}-2 a_{1}\right)-2 \sigma^{2} c_{1}, \\
& \widetilde{c}_{2}=c_{2}+\sigma\left(a_{1}-b_{2}\right)+\sigma^{2}\left(a_{2}-b_{1}\right)+\sigma^{3} c_{1} .
\end{aligned}
$$

By chosing $\sigma$ such that $\widetilde{c}_{2}=0$ and noting the fact that $c_{1}$ and $c_{2}$ have the same status, we can, in fact, get a similar situation as $c_{1}=0$.

Proof. (1) If $\operatorname{Rank}(\Xi)=0$, then $F_{2}(x) \equiv 0$; the result is trivial. If $\operatorname{Rank}(\Xi)=1$, there exist two constants $\sigma_{1}, \sigma_{2}$ such that $\sigma_{1}\left(a_{1}, b_{1}, c_{1}\right)+\sigma_{2}\left(a_{2}, b_{2}, c_{2}\right)=0$, which means $I(x)=\sigma_{1} x_{1}+$ $\sigma_{2} x_{2}$ is a first integral.

(2) If $\operatorname{Rank}(\Xi)=2$ and $c_{1}=a_{2}=0$, (16) can be represented as

$$
\begin{aligned}
& \dot{x}_{1}=a_{1} x_{1}^{2}+b_{1} x_{1} x_{2}, \\
& \dot{x}_{2}=b_{2} x_{1} x_{2}+c_{2} x_{1}^{2},
\end{aligned}
$$

whose integrability is equivalent to that of the system

$$
\begin{aligned}
& \dot{x}_{1}=a_{1} x_{1}+b_{1} x_{2}, \\
& \dot{x}_{2}=b_{2} x_{2}+c_{2} x_{1} .
\end{aligned}
$$

By Poincaré's result, system (16) is formally integrable if and only if two eigenvalues of the matrix $\left(\begin{array}{ll}a_{1} & b_{1} \\ c_{2} & b_{2}\end{array}\right)$ satisfy condition (3).
Now, if $\operatorname{Rank}(\Xi)=2$ and $c_{1}=0, a_{2} \neq 0,(16)$ can be represented as

$$
\begin{aligned}
& \dot{x}_{1}=a_{1} x_{1}^{2}+b_{1} x_{1} x_{2}, \\
& \dot{x}_{2}=a_{2} x_{2}^{2}+b_{2} x_{1} x_{2}+c_{2} x_{1}^{2} .
\end{aligned}
$$

By transformation (18), one can get

$$
\begin{aligned}
& \dot{y}_{1}=\tilde{a}_{1} y_{1}^{2}+\widetilde{b}_{1} y_{1} y_{2}, \\
& \dot{y}_{2}=\widetilde{a}_{2} y_{2}^{2}+\widetilde{b}_{2} y_{1} y_{2}+\widetilde{c}_{2} y_{1}^{2},
\end{aligned}
$$

with

$$
\begin{aligned}
& \widetilde{a}_{1}=a_{1}-\sigma b_{1}, \\
& \widetilde{b}_{1}=b_{1}, \\
& \widetilde{a}_{2}=a_{2}, \\
& \widetilde{b}_{2}=b_{2}+\sigma\left(b_{1}-2 a_{2}\right), \\
& \widetilde{c}_{2}=\left(a_{2}-b_{1}\right) \sigma^{2}+\left(a_{1}-b_{2}\right) \sigma+c_{2} .
\end{aligned}
$$

(3) If $a_{2}=b_{1}$ and $a_{1}=b_{2}$, without loss of generality, one can assume that $a_{1}=b_{2}=0$ after the same discussion as in Remark 4. Then (4) becomes

$$
\begin{aligned}
& \dot{x}_{1}=b_{1} x_{1} x_{2}, \\
& \dot{x}_{2}=a_{2} x_{2}^{2}+c_{2} x_{1}^{2},
\end{aligned}
$$

with $b_{1} a_{2} c_{2} \neq 0$. Assume $I_{l}(x)=\sum_{k=0}^{l} h_{k} x_{1}^{l-i} x_{2}^{i}$ is a first integral of system (26) with some integers $l>0, h_{k}(k=$ $0, \ldots, l)$ being the coefficients to be determined. Then we can get

$$
\begin{aligned}
0 \equiv & \left\langle\nabla_{x} I_{l}(x), F_{2}(x)\right\rangle \\
= & \sum_{k=0}^{l} k h_{k} \mathcal{c}_{2} x_{1}^{l-k+2} x_{2}^{k-1}+k h_{k} a_{2} x_{1}^{l-k} x_{2}^{k+1} \\
& +(l-k) h_{k} b_{1} x_{1}^{l-k} x_{2}^{k+1} .
\end{aligned}
$$

Comparing each term of the above equation, we can get

$$
\begin{gathered}
x_{1}^{l+1}: h_{1} c_{2}=0 ; \\
x_{1}^{l} x_{2}: 2 h_{2} c_{2}=-l h_{0} b_{1} ; \\
x_{1}^{l-1} x_{2}^{2}: 3 h_{3} c_{2}=-h_{1}\left(a_{2}+(l-1) b_{1}\right) ; \\
\vdots \\
x_{1}^{l-k} x_{2}^{k+1}:(k+2) h_{k+2} c_{2}=-h_{k}\left(k a_{2}+(l-k) b_{1}\right) ; \\
\vdots \\
x_{1}^{2} x_{2}^{l-1}: l h_{l} c_{2}=-h_{l-2}\left((l-2) a_{2}+2 b_{1}\right) ; \\
x_{1} x_{2}^{l}: 0=h_{l-1}\left((l-1) a_{2}+b_{1}\right) ; \\
x_{2}^{l+1}: 0=l h_{l} a_{2},
\end{gathered}
$$


which implies that the first integral $I_{l}(x)$ is nontrivial if and only if there exists an even integer $k_{0}, 0<k_{0}<l$, such that $k_{0} a_{2}+\left(l-k_{0}\right) b_{1}=0=l b_{1}$, which contradicts with the condition $b_{1}=a_{2} \neq 0$.

(4) If $\left(a_{2}-b_{1}\right)^{2}+\left(a_{1}-b_{2}\right)^{2} \neq 0$, without loss of generality, one can assume that $c_{2}=0$. Then (4) becomes

$$
\begin{aligned}
& \dot{x}_{1}=a_{1} x_{1}^{2}+b_{1} x_{1} x_{2}, \\
& \dot{x}_{2}=a_{2} x_{2}^{2}+b_{2} x_{1} x_{2},
\end{aligned}
$$

with $a_{2}\left(a_{1} b_{2}-a_{2} b_{1}\right) \neq 0$. Assume that $I_{l}(x)=\sum_{k=0}^{l} h_{k} x_{1}^{i} x_{2}^{l-i}$ is a first integral of system (26) with $l>0, h_{k}(k=0, \ldots, l)$ being the coefficients to be determined. Then we can get

$$
\begin{aligned}
0 \equiv & \left\langle\nabla_{x} I_{l}(x), F_{2}(x)\right\rangle \\
= & \sum_{k=0}^{l} h_{k}\left(k a_{1}+(l-k) b_{2}\right) x_{1}^{k+1} x_{2}^{l-k} \\
& \quad+h_{k}\left(k b_{1}+(l-k) a_{2}\right) x_{1}^{k} x_{2}^{l-k+1} .
\end{aligned}
$$

Equating each term of the above equation, one can get

$$
\begin{aligned}
& x_{2}^{l+1}: h_{0} l a_{2}=0 \\
& x_{1} x_{2}^{l}: h_{1}\left(b_{1}+(l-1) a_{2}\right)=-h_{0}\left(l b_{2}\right) ; \\
& x_{1}^{2} x_{2}^{l-1}: h_{2}\left(2 b_{1}+(l-2) a_{2}\right)=-h_{1}\left(a_{1}+(l-1) b_{2}\right) ; \\
& \vdots \\
& x_{1}^{k+1} x_{2}^{l-k}: h_{k}\left(k a_{1}+(l-k) b_{2}\right) \\
& =-h_{k-1}\left((k-1) b_{1}+(l-k+1) a_{2}\right) ; \\
& \quad \vdots \\
& x_{1}^{l-1} x_{2}^{2}: h_{l-1}\left((l-1) b_{1}+a_{2}\right) \\
& =-h_{l-2}\left((l-2) a_{1}+2 b_{2}\right) ; \\
& x_{1}^{l} x_{2}: h_{l} l b_{1}=-h_{l-1}\left((l-1) a_{1}+b_{2}\right) ; \\
& x_{1}^{l+1}: 0=l h_{l} a_{1} ;
\end{aligned}
$$

these equations imply that the first integral $I_{l}(x)$ is nontrivial if and only if there exist two integers $k_{1}, k_{2}, 0<k_{1} \leq k_{2} \leq l$, such that $k_{1} b_{1}+\left(l-k_{1}\right) a_{2}=0$ and $k_{2} a_{1}+\left(l-k_{2}\right) b_{2}=0$.

Then, based on the above discussion, one can summarize several necessary conditions of integrability for the original system (1).

Theorem 5. System (1) is formally integrable, if one of the following cases holds up to a equivalent transformation:

(1) $\operatorname{Rank}(\Xi) \leq 1$;

(2) $\operatorname{Rank}(\Xi)=2, c_{1}=a_{2}=0$, and the eigenvalues of the matrix $\left(\begin{array}{ll}a_{1} & b_{1} \\ c_{2} & b_{2}\end{array}\right)$ satisfy the condition (3);
(3) $\operatorname{Rank}(\Xi)=2, c_{1}=0, a_{2} \neq 0,\left(a_{2}-b_{1}\right)^{2}+\left(a_{1}-b_{2}\right)^{2} \neq 0$, and there exist three integers $k_{1}, k_{2}, l$, such that $0<$ $k_{1} \leq k_{2} \leq l, k_{1} b_{1}+\left(l-k_{1}\right) a_{2}=0$, and $k_{2} a_{1}+\left(l-k_{2}\right) b_{2}=$ 0 .

Example 6. Consider system

$$
\begin{aligned}
& \dot{x}_{1}=x_{1}^{2}+2 x_{1} x_{2}+3 x_{2}^{2}+f(x), \\
& \dot{x}_{2}=2 x_{2}^{2}+4 x_{1} x_{2}+g(x),
\end{aligned}
$$

where $f(x)=O\left(|x|^{3}\right)$ and $g(x)=O\left(|x|^{3}\right)$ are analytic in $x$. This is, in fact, a higher order perturbed system of a homogeneous quadratic vector field (Collins, [15]):

$$
\begin{aligned}
& \dot{x}_{1}=x_{1}^{2}+2 x_{1} x_{2}+3 x_{2}^{2}, \\
& \dot{x}_{2}=2 x_{2}^{2}+4 x_{1} x_{2} .
\end{aligned}
$$

Since $x_{1}$ and $x_{2}$ have the same status, therefore, (33) is indeed equivalent to a new homogeneous quadratic vector field with

$$
\Xi=\left(\begin{array}{lll}
a_{1} & b_{1} & c_{1} \\
c_{2} & b_{2} & a_{2}
\end{array}\right)=\left(\begin{array}{lll}
2 & 4 & 0 \\
3 & 2 & 1
\end{array}\right) .
$$

Then, by simple computation, one can get that $\operatorname{Rank}(\Xi)=2$, $c_{1}=0, a_{2} \neq 0$, and $\left(a_{2}-b_{1}\right)^{2}+\left(a_{1}-b_{2}\right)^{2}=(1-4)^{2}+(2-2)^{2}=$ $9 \neq 0$. Further, for any three integers $k_{1}, k_{2}, 0<k_{1} \leq k_{2} \leq l$, we have

$$
\begin{aligned}
& k_{1} b_{1}+\left(l-k_{1}\right) a_{2}=l+3 k_{1}>0, \\
& k_{2} a_{1}+\left(l-k_{2}\right) b_{2}=2 l>0 .
\end{aligned}
$$

By Theorems 3 and 2, both systems (33) and (32) do not have any nontrivial formal first integral; that is to say, they are not formally integrable.

\section{Higher Dimensional Systems with Invariant Manifolds}

Consider system (5), where $F(x, y)$ and $G(x, y)$ are analytic, $F(0,0)=0, G(0,0)=0$. Suppose (5) has an invariant manifold expressed as (6), where $h(x)$ is an analytic vectorvalued function.

In general, we say a function $\Phi(z)$ in Euclidean space $\mathbb{R}^{n}$ is independent from $m(1 \leq m<n)$-dimensional invariant manifold $\mathscr{M} \subset \mathbb{R}^{n}$, if $\Phi(z)$ 三 const. on $\mathscr{M}$. Then the first integrals of (5) can be divided into two classes by the independence from the manifold $\mathscr{Z}$. And it is easy to conclude that any first integral independent from $\mathscr{Z}$ also leads to a first integral of

$$
\dot{x}=F(x, h(x)) .
$$

Lemma 7. If $H(x, y)$ is a nontrivial formal first integral of system (5), then $\Phi(x)=H(x, h(x))$ is a formal first integral of the subsystem (36). Furthermore, if $H(x, y)$ is independent from $\mathscr{Z}, \Phi(x)=H(x, h(x))$ is also a nontrivial formal first integral of (36). 
Expanding $F(x, h(x))=F_{m}(x)+F_{m+1}(x)+O\left(|x|^{m+2}\right)$ with the lowest order terms $F_{m}(x)$, we have the following.

Lemma 8. If system (5) has a nontrivial formal first integral independent from $\mathscr{Z}$, then

$$
\dot{x}=F_{m}(x)
$$

has a nontrivial homogeneous first integral.

Proof. The proof is easy and can be found in [11], so we omit it here.

If $m=1, F_{m}(x)$ can be represented as $F_{m}(x)=C x$ ( $C$ is $d \times d$ matrix). If $m>1$, (37) can be treated as a quasihomogeneous system of degree $m$ with exponents $s_{1}=$ $s_{2}=\cdots=s_{d}=1$ (for more details, see [2,9]), we look for a balance $\xi$ of the given vector field $F_{m}(x)$ by solving the algebraic equations $(1 /(m-1)) \xi+F_{m}(\xi)=0$. Then one can make the change of variables $x=t^{1 /(1-m)}(\xi+u), t=\ln \tau$, such that (37) reads

$$
u^{\prime}=K u+\widetilde{F}(u)
$$

where the prime means the derivative with respect to $\tau$ and

$$
K=\frac{1}{m-1} E+\frac{\partial F_{m}}{\partial x}(\xi)
$$

is the so-called Kovalevskaya matrix associated with the balance $\xi, \widetilde{F}(u)=(1 /(m-1)) \xi+F_{m}(\xi+u)-\left(\partial F_{m} / \partial x\right)(\xi) u$.

Theorem 9. Assume $F(x, h(x))=F_{m}(x)+F_{m+1}(x)+O\left(|x|^{m+2}\right)$ with the lowest order terms $F_{m}(x)$.

(1) If $m=1$ and the eigenvalues $\rho_{1}, \ldots, \rho_{d}$ of the matrix $C$ are $\mathbb{N}$-independent, then (5) does not have any formal first integral independent from $\mathscr{Z}$.

(2) If $m>1$, for any balance $\xi$ of the vector field $F_{m}(x)$, the eigenvalues $\lambda_{1}, \ldots, \lambda_{d}$ of the Kovalevskaya matrix $K$ associated with the balance $\xi$ are $\mathbb{N}$-independent. Then (5) does not have any formal first integral independent from $\mathscr{Z}$.

Proof. (1) When $m=1$, if the eigenvalues $\rho_{1}, \ldots, \rho_{d}$ of the matrix $C$ are $\mathbb{N}$-independent, then, by Theorem $\mathrm{B}$ in [9], system (37) does not have any formal first integral independent from $\mathscr{Z}$. And (5) does not have any formal first integral independent from $\mathscr{Z}$.

(2) When $m>1$, if the eigenvalues $\lambda_{1}, \ldots, \lambda_{d}$ of the Kovalevskaya matrix $K$ associated with the balance $\xi$ are $\mathbb{N}$ independent, then, by Theorem $\mathrm{C}$ in [9], (37) does not have any formal first integral independent from $\mathscr{Z}$. On the other hand, if system (5) has a formal first integral independent from $\mathscr{Z}$, (36) has a nontrivial formal first integral. By Lemma 8, we know that (37) should have a homogeneous first integral. There comes a contradiction.
Example 10. Let us consider a three-dimensional system of Lotka-Volterra type

$$
\begin{aligned}
& \dot{x}_{1}=x_{1}\left(-a x_{3}\right), \\
& \dot{x}_{2}=x_{2}\left(c x_{1}+d x_{2}+e x_{3}\right), \\
& \dot{x}_{3}=x_{3}\left(1+b x_{1}+a x_{3}\right),
\end{aligned}
$$

where $a, b, c, d, e, f$ are real nonzero constants.

Theorem 11. Assume that ae $<0$ or $a$ and $e$ are $\mathbb{N}$ independent. Then system (40) has only one analytic first integral $\Phi\left(x_{1}, x_{2}, x_{3}\right)=a x_{1} x_{3}+(1 / 2) b x_{1}^{2}+x_{1}$, in sense of functional independence.

Proof. Firstly, it is easily shown that $\Phi\left(x_{1}, x_{2}, x_{3}\right)=a x_{1} x_{3}+$ $(1 / 2) b x_{1}^{2}+x_{1}$ is an analytic first integral of (40) indeed. Next, we will prove that system (40) does not have any other formal first integral independent from $\Phi\left(x_{1}, x_{2}, x_{3}\right)$.

One can find that (40) has an invariant manifold

$$
\mathscr{Z}_{0}=\left\{\left(x_{1}, x_{2}, x_{3}\right) \mid x_{3}=-\frac{1}{a}-\frac{b}{2 a} x_{1}\right\} ;
$$

then it can be reduced to

$$
\begin{aligned}
\left(\begin{array}{l}
\dot{x}_{1} \\
\dot{x}_{2}
\end{array}\right)= & \left(\begin{array}{cc}
1 & 0 \\
0 & -\frac{e}{a}
\end{array}\right)\left(\begin{array}{l}
x_{1} \\
x_{2}
\end{array}\right) \\
& +\left(\begin{array}{c}
\frac{b}{2} x_{1}^{2} \\
\left(c-\frac{b e}{2 a}\right) x_{1} x_{2}+d x_{2}^{2}
\end{array}\right) .
\end{aligned}
$$

Denote

$$
\begin{aligned}
B & =\left(\begin{array}{cc}
1 & 0 \\
0 & -\frac{e}{a}
\end{array}\right), \\
F_{2}(x) & =\left(\begin{array}{c}
\frac{b}{2} x_{1}^{2} \\
\left(c-\frac{b e}{2 a}\right) x_{1} x_{2}+d x_{2}^{2}
\end{array}\right) .
\end{aligned}
$$

Since $a e<0$ or $a$ and $e$ are $\mathbb{N}$-independent, then the eigenvalues of $B$ are $\mathbb{N}$-independent. From the case of $m=$ 1 of Theorem 9, we know that system (40) does not have any other formal first integral independent from $\mathscr{Z}_{0}$, which implies that if (40) has another nontrivial formal first integral $I\left(x_{1}, x_{2}, x_{3}\right)$ functionally independent from $\Phi\left(x_{1}, x_{2}, x_{3}\right)=$ $a x_{1} x_{3}+(1 / 2) b x_{1}^{2}+x_{1}$, without loss of generality, $I\left(x_{1}, x_{2}, x_{3}\right)$ can be expressed as $I\left(x_{1}, x_{2}, x_{3}\right)=\left(a x_{3}+(1 / 2) b x_{1}+\right.$ $1)^{l} \phi\left(x_{1}, x_{2}, x_{3}\right)$ with some integer $l$ and formal function $\phi\left(x_{1}, x_{2}, x_{3}\right)$ which can not be divided exactly by $a x_{3}+$ $(1 / 2) b x_{1}+1$.

On the other hand, note that (40) has another invariant manifold

$$
\mathscr{Z}_{1}=\left\{\left(x_{1}, x_{2}, x_{3}\right) \mid x_{1}=0\right\} \text {; }
$$


then (40) can be reduced to

$$
\begin{aligned}
& \dot{x}_{2}=x_{2}\left(d x_{2}+e x_{3}\right), \\
& \dot{x}_{3}=x_{3}\left(1+a x_{3}\right) .
\end{aligned}
$$

By simple calculation, it is not difficult to get that (45) does not have any formal first integrals, which means $I\left(x_{1}, x_{2}, x_{3}\right)$ can be further expressed as $I\left(x_{1}, x_{2}, x_{3}\right)=\left(a x_{3}+(1 / 2) b x_{1}+\right.$ $1)^{l} x_{1}^{m} \tilde{\phi}\left(x_{1}, x_{2}, x_{3}\right)$ with some integer $m$ and formal function $\widetilde{\phi}\left(x_{1}, x_{2}, x_{3}\right)$ which can not be divided exactly by $x_{1}$.

Without loss of generality, let $l \geq m$; then

$$
\begin{aligned}
I\left(x_{1}, x_{2}, x_{3}\right)= & \left(a x_{3}+\frac{1}{2} b x_{1}+1\right)^{l-m} \Phi\left(x_{1}, x_{2}, x_{3}\right)^{m} \\
& \cdot \widetilde{\phi}\left(x_{1}, x_{2}, x_{3}\right)
\end{aligned}
$$

this implies that $\bar{I}\left(x_{1}, x_{2}, x_{3}\right)=\left(a x_{3}+(1 / 2) b x_{1}+1\right)^{l-m} \widetilde{\phi}\left(x_{1}\right.$, $\left.x_{2}, x_{3}\right)$ is also a formal first integral of (40) and independent from $\Phi\left(x_{1}, x_{2}, x_{3}\right)$. However, from the above discussions, one can easily find the contradiction. The proof is completed.

We remark that a large number of systems in applications can be viewed as the objects with center manifolds. In what follows, we consider this class of systems

$$
\begin{aligned}
& \dot{x}=A x+f(x, y), \\
& \dot{y}=B y+g(x, y),
\end{aligned}
$$

where $(x, y) \in \mathbb{R}^{d} \times \mathbb{R}^{s}, f(x, y)=O\left(|x, y|^{2}\right), g(x, y)=$ $O\left(|x, y|^{2}\right)$, the eigenvalues $\sigma_{1}, \ldots, \sigma_{d}$ of the matrix $A$ have zero real parts, and the eigenvalues $\lambda_{1}, \ldots, \lambda_{s}$ of the matrix $B$ have nonzero real parts.

We are now going to investigate some new criteria for nonexistence of formal integrals for system (47) by combining Theorem 9 with the center manifold theory [14]. In fact, by center manifold theorem, (47) has a center manifold in a neighborhood of the original point, which can be locally represented as

$$
\begin{aligned}
& \mathscr{Z}^{c}(0)=\left\{(x, y) \in \mathbb{R}^{d} \times \mathbb{R}^{s}|y=h(x),| x \mid\right. \\
& <\delta, h(0)=0, \operatorname{Dh}(0)=0\},
\end{aligned}
$$

with $\delta$ being sufficiently small, and $h(x)$ satisfies equation

$$
D h(x)[A x+f(x, h(x))]=B h(x)+g(x, h(x))
$$

or

$$
\begin{aligned}
\mathcal{N}(h(x)) \equiv & D h(x)[A x+f(x, h(x))]-B h(x) \\
& -g(x, h(x))=0 .
\end{aligned}
$$

The following theorem allows us to compute the local center manifold to any desired degree of accuracy by solving (49) to the same degree of accuracy. Therefore it also guarantees the effectiveness of our discussions in the latter part of this section.
Theorem 12. Let $\phi: \mathbb{R}^{d} \rightarrow \mathbb{R}^{s}$ be a $\mathbf{C}^{1}$ mapping with $\phi(0)=$ $D \phi(0)=0$ such that $\mathcal{N}(\phi(x))=O\left(|x|^{q}\right)$ as $x \rightarrow 0$ for some $q>1$. Then

$$
|h(x)-\phi(x)|=O\left(|x|^{q}\right) \quad \text { as } x \longrightarrow 0 .
$$

Proof. See [16].

On the center manifold $\mathscr{Z}^{c}(0)$, system (47) can be reduced to

$$
\dot{x}=A x+f(x, h(x)) .
$$

Expanding $f(x, h(x))=f_{l}(x)+f_{l+1}(x)+O\left(|x|^{l+2}\right)$ with the lowest order terms $f_{l}(x)$, then, for the case $A=O$, with the same idea as in the above section, one can consider the corresponding homogeneous system

$$
\dot{x}=f_{l}(x) \text {. }
$$

And we also treat it as a quasihomogeneous system of degree $l$ with exponents $s_{1}=s_{2}=\cdots=s_{d}=1, l>1$.

Assuming the algebraic equation $(1 /(l-1)) \eta+f_{l}(\eta)=0$ has a nonzero solution $\eta \in \mathbb{C}^{d}$, one can get the corresponding Kovalevskaya matrix

$$
K=\frac{1}{l-1} E+\frac{\partial f_{l}}{\partial x}(\eta)
$$

Then we have the following results.

Theorem 13. (1) If the eigenvalues $\sigma_{1}, \ldots, \sigma_{d}$ of the matrix $A$ are $\mathbb{N}$-independent, then system (47) does not have any formal first integral independent from $\mathscr{Z}^{c}(0)$.

(2) If $A=O$ and, for any balance $\xi$ of the vector field $f_{l}(x)$, the eigenvalues $\rho_{1}, \ldots, \rho_{d}$ of the Kovalevskaya matrix $K$ associated with $\xi$ are $\mathbb{N}$-independent, then system (47) does not have any formal first integral independent from $\mathscr{Z}^{c}(0)$.

(3) If the eigenvalues $\lambda_{1}, \ldots, \lambda_{s}$ of the matrix $B$ are $\mathbb{N}$ independent, and one of the above two cases holds, then system (47) does not have any nontrivial formal first integral.

Proof. (1) From Theorem B in [9], if the eigenvalues $\sigma_{1}, \ldots, \sigma_{d}$ of $A$ are $\mathbb{N}$-independent, then system (52) does not have any formal first integrals. Hence it is easy to prove that system (47) does not have any formal first integrals independent from $\mathscr{Z}^{c}(0)$; we get the first result.

(2) If $A=O$ and the eigenvalues $\rho_{1}, \ldots, \rho_{d}$ of the Kovalevskaya matrix $K$ associated with the balance $\xi$ are $\mathbb{N}$-independent, from Theorem $C$ in [9], we can get the nonexistence of formal first integrals for system (53); then (47) does not have any formal first integrals independent from $\mathscr{Z}^{c}(0)$; the second result is obtained.

(3) If both eigenvalues $\sigma_{1}, \ldots, \sigma_{d}$ of the matrix $A$ and the ones $\lambda_{1}, \ldots, \lambda_{s}$ of the matrix $B$ are $\mathbb{N}$-independent, respectively, by the assumptions for system (47), all the eigenvalues $\sigma_{1}, \ldots, \sigma_{d}, \lambda_{1}, \ldots, \lambda_{s}$ are, in fact, $\mathbb{N}$-independent; then, by Theorem B in [9], it is obvious that (47) does not have any formal first integrals.

At last, assume the eigenvalues $\lambda_{1}, \ldots, \lambda_{s}$ of the matrix $B$ are $\mathbb{N}$-independent and the second condition holds. If $I(x, y)$ 
is a formal first integral of (47), we claim that $I(x, y)$ is trivial. Otherwise, expanding $I(x, y)=I_{l}(x, y)+I_{l+1}(x, y)+\cdots$ with the lowest order terms $I_{l}(x, y)$ of order $l>0$, we have

$$
\begin{aligned}
0 \equiv & \left\langle\frac{\partial}{\partial x} I(x, y), f(x, y)\right\rangle \\
& +\left\langle\frac{\partial}{\partial y} I(x, y), B y+g(x, y)\right\rangle \\
= & \left\langle\frac{\partial}{\partial y} l_{l}(x, y), B y\right\rangle+\text { h.o.t }
\end{aligned}
$$

where h.o.t denotes the higher order terms. Without loss of generality, we can assume that $B=\operatorname{diag}\left\{\lambda_{1}, \ldots, \lambda_{s}\right\}$. Equating the same order of $(x, y)$ in (55), we have

$$
\begin{aligned}
0 & \equiv\left\langle\frac{\partial}{\partial y} l_{l}(x, y), B y\right\rangle \\
& =\sum_{\alpha \in \mathbb{Z}^{d}, \beta \in \mathbb{Z}^{s},|\alpha|+|\beta|=l} I_{\alpha \beta}\left(\beta_{1} \lambda_{1}+\cdots+\beta_{s} \lambda_{s}\right) x^{\alpha} y^{\beta} .
\end{aligned}
$$

Then, on the one hand, since $\lambda_{1}, \ldots, \lambda_{s}$ are $\mathbb{N}$-independent, it is not difficult to see that $I_{\alpha \beta}=0$ for all nonzero $\beta \in \mathbb{Z}^{s},|\beta| \leq$ l. On the other hand, from the discussions in the second case, $I(x, y)$ must be dependent on $\mathscr{Z}^{c}(0)$; that is,

$$
I(x, h(x)) \equiv \text { const. }
$$

which implies that $I_{l}$ must contain $y$; there is a contradiction. The proof is completed.

Example 14. Consider the following system:

$$
\begin{aligned}
& \dot{x}_{1}=\alpha x_{2} y+\beta x_{1} y, \\
& \dot{x}_{2}=x_{1} y+x_{2}^{3}, \\
& \dot{y}=-y+x_{1} x_{2},
\end{aligned}
$$

$$
\left(x_{1}, x_{2}, y\right) \in \mathbb{R}^{3}
$$

where $\alpha, \beta$ are parameters.

By simple computation, we can get that system (58) has a center manifold which can locally be represented as follows:

$$
\begin{aligned}
& \mathscr{Z}^{c}(0)=\left\{\left(x_{1}, x_{2}, y\right) \in \mathbb{R}^{3} \mid y=x_{1} x_{2}\right. \\
& \left.\quad+O\left(x^{4}\right),\left|x_{1}, x_{2}\right|<\delta\right\},
\end{aligned}
$$

with $\delta$ sufficiently small. have

Restricting system (58) to the center manifold $\mathscr{Z}^{c}(0)$, we

$$
\begin{aligned}
& \dot{x}_{1}=\alpha x_{1} x_{2}^{2}+\beta x_{1}^{2} x_{2}, \\
& \dot{x}_{2}=x_{1}^{2} x_{2}+x_{2}^{3},
\end{aligned}
$$

which can be treated as a quasihomogeneous system of degree 3 with exponents $s_{1}=s_{2}=1$. So $m=3, S=E=\operatorname{diag}(1,1)$,
$H=(1 /(m-1)) S=\operatorname{diag}(1 / 2,1 / 2)$. Suppose $(60)$ has a particular solution of the form

$$
x(t)=t^{-H} \xi .
$$

Then $\xi=\left(\xi_{1}, \xi_{2}\right)$ has to satisfy the algebraic equation as follows:

$$
\frac{1}{2}\left(\begin{array}{c}
\xi_{1} \\
\xi_{2}
\end{array}\right)+\left(\begin{array}{c}
\alpha \xi_{1} \xi_{2}^{2}+\beta \xi_{1}^{2} \xi_{2} \\
\xi_{1}^{2} \xi_{2}+\xi_{2}^{3}
\end{array}\right)=0
$$

which has four nontrivial solutions,

$$
\begin{gathered}
\left(0, \pm \frac{1}{\sqrt{-2}}\right) \\
( \pm e, \pm a e)
\end{gathered}
$$

with $a=\left(\beta \pm \sqrt{\beta^{2}+4(\alpha-1)}\right) / 2, e=1 / \sqrt{-2(\alpha+a \beta)}$. And the corresponding Kovalevskaya matrices are, respectively,

$$
\begin{gathered}
\left(\begin{array}{cc}
\frac{1-\alpha}{2} & 0 \\
0 & -\frac{1}{2}
\end{array}\right), \\
\left(\begin{array}{cc}
\alpha \beta & 2 a(\alpha+a \beta) \\
2 a & 2
\end{array}\right) e^{2},
\end{gathered}
$$

and the eigenvalues are

$$
\begin{gathered}
\left\{-\frac{1}{2}, \frac{1}{2}-\frac{\alpha}{2}\right\}, \\
\left\{\frac{A+B}{2} e^{2}, \frac{A-B}{2} e^{2}\right\},
\end{gathered}
$$$$
\text { respectively; here } A=2+a \beta, B=
$$
$\sqrt{(2+a \beta)^{2}-8 a^{2}(2 \alpha+a \beta)}$.

Corollary 15. System (58) does not have any formal first integral, if

(1) $\alpha$ is irrational;

(2) $A \neq 0=B$;

(3) $A$ and $B$ are $\mathbb{N}$-independent.

Proof. We only prove Cases (2) and (3), the proof for case (1) is similar. Denote

$$
\begin{aligned}
& \lambda_{1}=\frac{A+B}{2} e^{2}, \\
& \lambda_{2}=\frac{A-B}{2} e^{2} .
\end{aligned}
$$

Then, by the given conditions, for any $k_{1}, k_{2} \in \mathbb{N}$,

$$
k_{1} \lambda_{1}+k_{2} \lambda_{2}=\left(\frac{k_{1}+k_{2}}{2} A+\frac{k_{1}-k_{2}}{2} B\right) e \xi_{2}^{2} \neq 0 .
$$

This implies that the eigenvalues $\lambda_{1}, \lambda_{2}$ of the Kovalevskaya matrix associated with the balance $( \pm e, \pm a e)$ are $\mathbb{N}$ independent. By Theorem 13, system (58) does not have any formal first integral. 


\section{Competing Interests}

The authors declare that they have no competing interests.

\section{Acknowledgments}

This work is supported by NSF (11301210 and 11226157) of China, National 973 project (2012CB821200) of China, NSF (20140520053JH) of Jilin, China, Postdoctoral Science Foundation (2014M560038) of China, Education Department Talents Project of Liaoning (LJQ2015029), Science and Technology Department Foundation of Liaoning (2015020021), Education Department Foundation of Liaoning (L2014549), and Fundamental Research Funds for the Central Universities (DC201502050304, DC201502050409, and DC201501043).

\section{References}

[1] V. V. Kozlov, Symmetries, Topology, and Resonances in Hamiltonian Mechanics, Springer, Berlin, Germany, 1995.

[2] S. D. Furta, "On non-integrability of general systems of differential equations," Zeitschrift für Angewandte Mathematik und Physik, vol. 47, no. 1, pp. 112-131, 1996.

[3] M. H. Poincaré, "Sur L'intégration algébrique des équations différentielles du premier ordre et du premier degré," Rendiconti del Circolo Matematico di Palermo, vol. 11, no. 1, pp. 193-239, 1897.

[4] A. Goriely, "Integrability, partial integrability, and nonintegrability for systems of ordinary differential equations," Journal of Mathematical Physics, vol. 37, no. 4, pp. 1871-1893, 1996.

[5] J. J. Morales Ruiz, Differencial Galois Theory and NonIntegrability of Hamiltonian Systems, Birkhäuser, Basel, Switzerland, 1999.

[6] H. Yoshida, "Necessary condition for the existence of algebraic first integrals. I. Kowalevski's exponents," Celestial Mechanics, vol. 31, no. 4, pp. 363-379, 1983.

[7] S. L. Ziglin, "Branching of solutions and nonexistence of first integrals in Hamiltonian mechanics. I," Functional Analysis and Its Applications, vol. 16, no. 3, pp. 181-189, 1982.

[8] S. L. Ziglin, "Branching of solutions and the nonexistence of first integrals in Hamiltonian mechanics. II," Functional Analysis and Its Applications, vol. 17, no. 1, pp. 6-17, 1983.

[9] S. Shi and Y. Li, "Non-integrability for general nonlinear systems," Zeitschrift für Angewandte Mathematik und Physik, vol. 52, no. 2, pp. 191-200, 2001.

[10] W. Li, Z. Xu, and S. Shi, "Nonexistence of formal first integrals for nonlinear systems under the case of resonance," Journal of Mathematical Physics, vol. 51, no. 2, Article ID 022703, 11 pages, 2010.

[11] F. Liu, S. Shi, and Z. Xu, "Nonexistence of formal first integrals for general nonlinear systems under resonance," Journal of Mathematical Analysis and Applications, vol. 363, no. 1, pp. 214219, 2010.

[12] S. Shi, "On the nonexistence of rational first integrals for nonlinear systems and semiquasihomogeneous systems," Journal of Mathematical Analysis and Applications, vol. 335, no. 1, pp. 125134, 2007.

[13] A. Goriely, Integrability and Non-Integrability of Dynamical Systems, World Scientific, Singapore, 2001.
[14] S. Wiggins, Introduction to Applied Nonlinear Dynamical Systems and Chaos, vol. 2 of Texts in Applied Mathematics, Springer, New York, NY, USA, 1990.

[15] C. B. Collins, "Algebraic conditions for a centre or a focus in some simple systems of arbitrary degree," Journal of Mathematical Analysis and Applications, vol. 195, no. 3, pp. 719-735, 1995.

[16] J. Carr, Applications of Centre Manifold Theory, vol. 35 of Applied Mathematical Sciences, Springer, New York, NY, USA, 1981. 


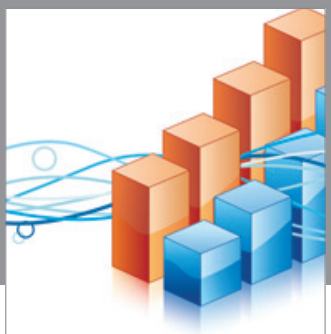

Advances in

Operations Research

vatem alat4

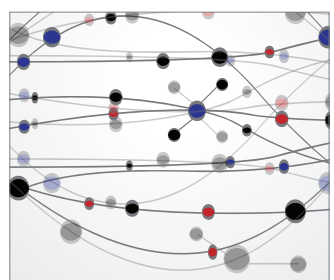

\section{The Scientific} World Journal
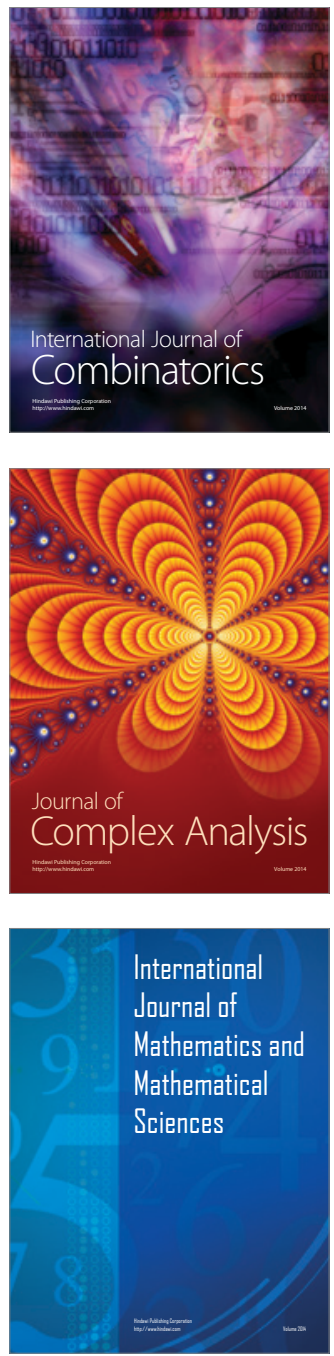
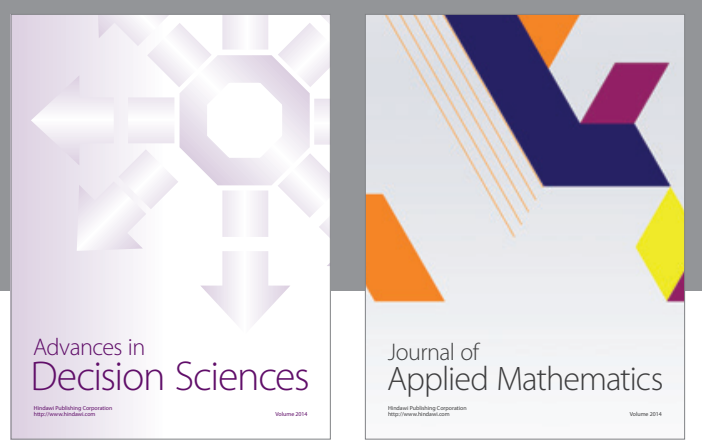

Algebra

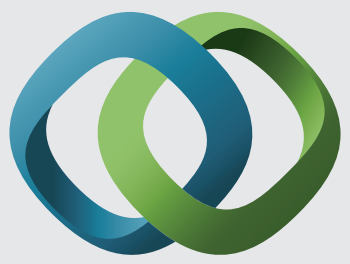

\section{Hindawi}

Submit your manuscripts at

http://www.hindawi.com
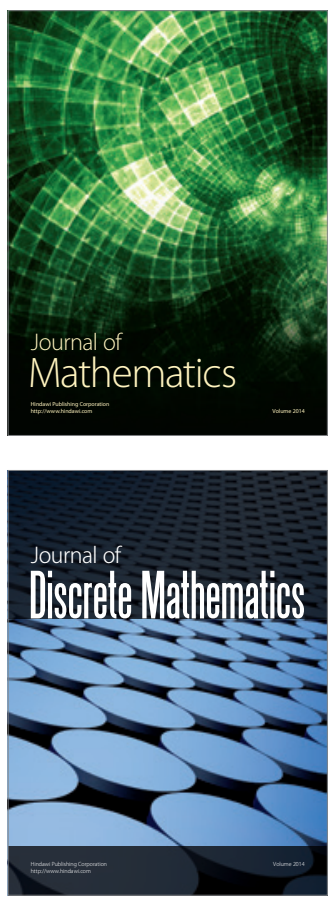

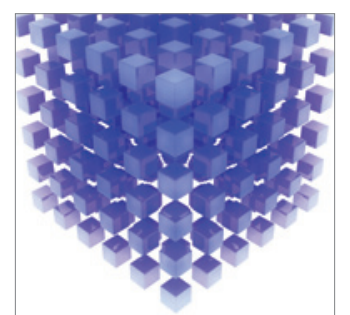

Mathematical Problems in Engineering
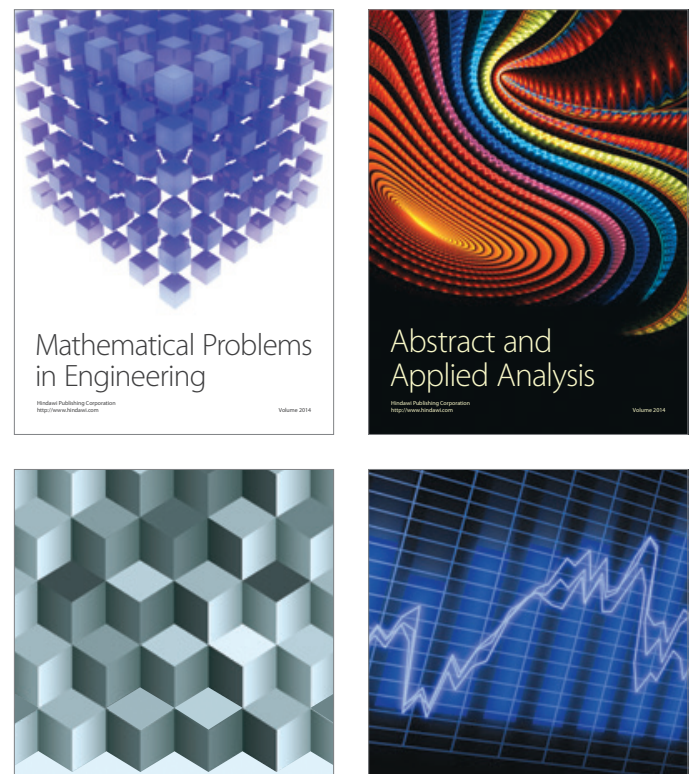

Journal of

Function Spaces

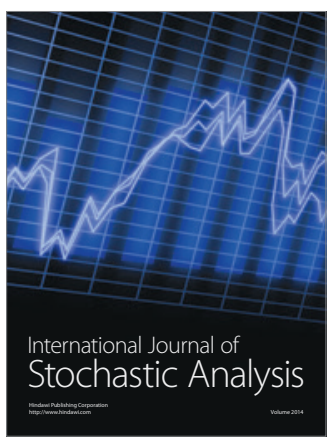

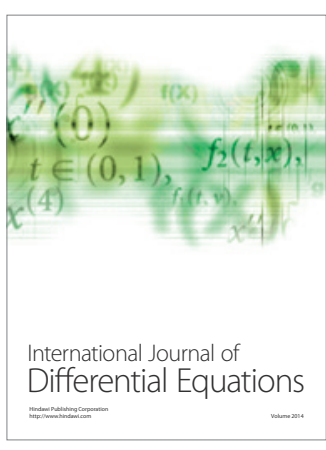
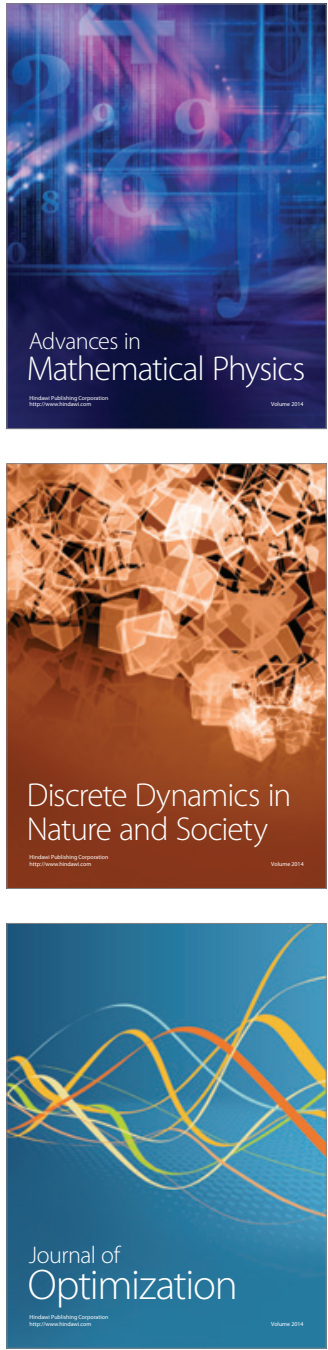